\title{
UNA REVISIÓN ANALÍTICA SOBRE EL PAPEL DE LA TIERRA EN LA TEORÍA ECONÓMICA DE DAVID RICARDO*
}

\author{
FRANCISCO CORREA RESTREPO** \\ UNIVERSIDAD AUTÓNOMA LATINOAMERICANA
}

Recibido/ Received/ Recebido: 08/10/2013 - Aceptado/ Accepted / Aprovado: 09/05/2014

\begin{abstract}
Resumen
Este artículo tiene como objetivo central analizar el papel del recurso tierra en la teoría económica de David Ricardo como primer acercamiento al análisis económico de los recursos naturales. Esto se realiza mediante una revisión bibliográfica analítica de la obra de Ricardo y de otros autores en torno a la misma. En este sentido, se examina el concepto de renta de la tierra, así como los planteamientos en torno a la llamada ley de los rendimientos decrecientes. Asimismo, se establecen algunas reflexiones críticas con relación al enfoque de la renta de la tierra. Un resultado importante del trabajo es que en el análisis de la renta de Ricardo está ausente el concepto de costo de oportunidad, lo cual implica una reconsideración del concepto de renta para abordar el análisis económico de recursos naturales.
\end{abstract}

Palabras clave: Economía ambiental, Teoría económica ricardiana, Renta de la tierra, Ley de los rendimientos decrecientes, Agricultura.

\section{AN ANALYTICAL REVIEW ABOUT THE ROLE OF LAND IN THE ECONOMIC THEORY OF DAVID RICARDO}

\begin{abstract}
The main objective of this paper is to analyze the resource of land from the economic theory of David Ricardo as a first approach to the economic analysis of natural resources. This is done through an analytic review of the literature on the work of Ricardo and other authors that have covered this subject. In this sense, the concept of ground rent is examined as well as the approaches around the so-called law of diminishing returns. Also, some critical thoughts are established about the approach to land rent. The results show that the concept of opportunity cost is absent from Ricardo's work on the income of land which implies a conceptual reconsideration on the income, in order to encompass the economic analysis on natural resources.

Keywords: Environmental economy, Economic theory of Ricardo, Income of the land, Law of diminishing returns, Agriculture.

* Artículo de reflexión generado a partir del proyecto de investigación titulado "Análisis del concepto del desarrollo sostenible desde las perspectivas de la economía ambiental y la economía ecológica", ejecutado en el período febrero- diciembre de 2013, financiado por la Universidad Autónoma Latinoamericana - UNAULA, Medellín (Colombia).

*: Economista y especialista en Evaluación Socioeconómica de Proyectos, Universidad de Antioquia. Magíster en Ciencias Económicas, Área de Economía de la Energía y los Recursos Naturales, Universidad Nacional de Colombia. Estudiante del Ph.D en Economía, SMC University (Suiza). Miembro del Grupo de Investigaciones Económicas GINVECO. Profesor de la cátedra de Economía Ambiental en la Universidad Autónoma Latinoamericana, Medellín (Colombia). Correo electrónico: francisco.correare@unaula.edu.co. Tel: (57) (4) -511-21-99. Dirección postal: Cra. 55A \# 49-51, Medellín.
\end{abstract}




\title{
UMA REVISÃO ANALÍTICA SOBRE O PAPEL DA TERRA NA TEORIA ECONÔMICA DE DAVID RICARDO
}

\begin{abstract}
Resumo
Este artigo tem como objetivo central analisar o papel do recurso terra na teoria econômica de David Ricardo como primeira aproximação à análise econômica dos recursos naturais. Isto se realiza mediante uma revisão bibliográfica analítica da obra de Ricardo e de outros autores em torno da mesma. Neste sentido, examina-se o conceito de renda da terra, bem como as propostas em torno da chamada lei dos rendimentos decrescentes. Também se estabelecem algumas reflexões críticas com relação ao enfoque da renda da terra. Um resultado importante do trabalho é que na análise da renda de Ricardo está ausente o conceito de custo de oportunidade, o qual implica uma reconsideração do conceito de renda para abordar a análise econômica de recursos naturais.

Palavras chave: Economia ambiental, Teoria econômica ricardiana, Renda da terra, Lei dos rendimentos decrescentes, Agricultura.

Correa, F. (2015). Una revisión analítica sobre el papel de la tierra en la teoría económica de David Ricardo. En: Revista de la Facultad de Ciencias Económicas de la Universidad Militar Nueva Granada. rev.fac.cienc.econ, XXIII (1).
\end{abstract}

JEL: B12.

\section{Introducción}

Es común localizar el surgimiento de la economía ambiental en la década de 1950. En particular, desde la literatura se asocia el origen de la economía de recursos naturales con la fundación de la organización Resources for the Future, en Estados Unidos, en 1952 (Pearce, 2002, 42). Ahora, la economía ambiental es el resultado del esfuerzo de la economía neoclásica por incorporar en su análisis el medio ambiente como objeto de estudio. Esta área de la economía utiliza, en esencia, los mismos conceptos y supuestos de la teoría neoclásica. De esta forma, la economía ambiental analiza el problema de la utilización del medio ambiente y los recursos naturales a partir del instrumental de la teoría económica tradicional. Desde esta área de la ciencia económica, el medio ambiente y los recursos naturales son tratados, al igual que los bienes económicos, como bienes escasos.

Ahora, si bien el análisis económico del medioambiente y los recursos naturales nació formalmente hace cerca de 60 años, la primera consideración de conceptos económicos relacionados con los recursos naturales y, en general, con la naturaleza es bastante antigua. De hecho, Alfranca $(2012,79)$ sitúa estas primeras consideraciones en la ética de Nicómaco de Aristóteles. Sin embargo, son los economistas clásicos quienes plantearon por primera vez, desde su análisis económico el funcionamiento de la economía en un mundo finito, es decir, en un mundo con limitaciones. En este sentido, tanto la teoría de la población de Malthus, como la teoría de los rendimientos decrecientes de la tierra de Ricardo, apuntan al límite ambiental que implicaría una oferta limitada de tierras de buena calidad. De otro lado, desde la literatura de la economía ambiental y la economía ecológica se señala la gran influencia que ha ejercido tanto la teoría de la renta como la ley de los rendimientos decrecientes de Ricardo en la economía ambiental. Por tanto, este artículo tiene como objetivo desarrollar una revisión analítica de la relación naturaleza-actividad económica (en particular el papel de la tierra en el sistema económico) en el modelo económico de David Ricardo.

Así, en la primera sección de este trabajo se establece brevemente los antecedentes teóricos e históricos de la obra de David Ricardo. La segunda sección se 
presenta la teoría de la renta de Ricardo y establece algunos puntos críticos de dicha teoría, lo cual es necesario para extender el enfoque ricardiano al análisis económico de los recursos naturales. Por su parte, la tercera analiza la llamada ley de los rendimientos decreciente de la tierra. En este sentido, la cuarta sección aporta algunos elementos de discusión con respecto a la relación entre la ley de los rendimientos decrecientes de la tierra y el problema poblacional. Por último, la quinta sección presenta las principales conclusiones del trabajo.

\section{Antecedentes}

Dentro del pensamiento clásico la obra de David Ricardo se destaca por establecer el primer modelo formal del funcionamiento del sistema económico. Para Hahne-Rima $(1995,143)$ las contribuciones de Ricardo muestran la transformación económica de Inglaterra durante cincuenta años después de que apareciera la riqueza de las naciones. Para esta época Inglaterra producía lo suficiente para abastecer los mercados de alimentos e, incluso, exportar a otras naciones de Europa, pero también la población estaba creciendo considerablemente y ya el país estaba experimentando la disminución de los rendimientos de la tierra. Sin embargo, prevalecían las medidas proteccionistas bajo la presión de los propietarios de tierras, a pesar de la necesidad creciente de establecer el libre comercio. Ahora, la obra central de Ricardo, publicada en 1817 bajo el nombre de Principios de economía política y tributación, recoge el aporte al análisis económico de este autor dentro de la escuela clásica y sintetiza sus principales ideas en torno a su gran preocupación: el progreso económico.

Se afirma que, al igual que Adam Smith, Ricardo considera la libertad económica como un factor fundamental para lograr el máximo de beneficio económico (Alfranca, 2012, 83). Para este autor, la base del crecimiento económico es la acumulación de capital y como ésta depende del beneficio económico, es claro que la obtención del mayor beneficio económico es esencial para el progreso económico de la sociedad. Ahora, Ricardo en su análisis económico presenta un modelo donde establece el proceso por el cual la actividad económica se vincula con la naturaleza, pero no porque haya habido en él preocupación por el deterioro del medio ambiente o la supervivencia del hombre, sino en parte porque su deseo era justificar por qué los terratenientes recibían una renta por la propiedad de la tierra (Costanza, Cumberland, Daly, Goodland \& Norgaard, $1999,30)^{1}$. No obstante, dentro de la teoría económica clásica, el análisis económico de la renta de la tierra es uno de los aportes más importantes de este autor. Si bien no fue el primero en establecer una teoría de la renta, sí presentó una explicación más sistemática y rigurosa del surgimiento de la renta en la economía. Además, a pesar de que en la obra de Adam Smith ya hay un esbozo de la existencia de las rentas diferenciales, Ricardo fue el primero en establecer analíticamente la ley de los rendimientos marginales decrecientes de la tierra. Dada la influencia que ha ejercido tanto la teoría de la renta como la ley de los rendimientos decrecientes de Ricardo en la economía ambiental, en las siguientes secciones se presentará una síntesis de tales aportes teóricos $y$, de igual manera, se plantearán algunos elementos de discusión crítica.

\section{La teoría ricardiana de la renta de la tierra}

\subsection{El concepto de renta en Ricardo}

En Inglaterra, como en otros países, hasta la revolución industrial la tierra representaba un poder económico y político. Explicar la naturaleza de ese poder económico era una de las preocupaciones centrales incluidas en la agenda de los teóricos de la economía clásica, partiendo por Adam Smith en 1776 (Hartwick, 1997). Así, en los primeros planteamientos de la ciencia económica la renta fue distinguida de otras formas de ingresos como el retorno periódico proveniente del uso de la tierra. Ya que la tierra en sí misma fue considerada como uno de los grandes agentes de la producción, la existencia de un tipo

1 Para los autores Costanza et al. (1999), la preocupación de Ricardo giraba al alrededor del límite impuesto por la oferta fija de recursos naturales y las posibilidades de crecimiento económico continuo. 
particular de ingreso atribuible a ella pareció un artificio particularmente adecuado (Gray, 1914, 466). Para Gray (1914) esta correlación completa entre la renta como un ingreso y la tierra como su fuente no estaba destinada a continuar. En la Inglaterra de la escuela clásica la renta era tomada de forma usual como una forma de ingresos que parecía dejar la base de los ingresos intacta. Según esta concepción teórica, año tras año, el terrateniente puede recibir un retorno sustancial sin disminuir el valor del capital de su inversión. Así, no es extraño que la incorruptibilidad de la base de la renta llegara a ser considerada una característica esencial de la renta como una forma de ingreso. De esta forma, resulto necesario definir una nueva base de la renta de modo que pudiera ajustarse a esta característica especial preconcebida de renta misma.

De acuerdo con esto, Ricardo (1993) modificó el concepto económico de la tierra y la definió como la fuente de un pago de renta. David Ricardo, en el capítulo II del libro principios de economía política y tributación, recoge sus ideas sobre la teoría de la renta. Allí explica el por qué la apropiación de la tierra, y con ella la creación de la renta, ocasionan variaciones en el valor relativo de los bienes, sin importar la cantidad de trabajo que se incluya en ellos (Ricardo, 1993, 51). En primer lugar, este autor define renta como "aquella parte del producto de la tierra que se paga al terrateniente por el uso de las fuerzas indestructibles del suelo" (Ricardo, 1993, 51). Ahora una clara distinción que hace Ricardo $(1993,50)$ es que si bien, ante la existencia de dos tierras vecinas con igual dimensión y misma fertilidad natural, pero sólo una de ellas dotada de mejor infraestructura (abono, drenaje, vallas y cercas), la remuneración que ambas tierras reciben por su uso se llama renta, pero es la tierra mejor equipada la que recibirá mayor remuneración. Ricardo $(1993,51)$ afirma que "sólo una parte de esa remuneración o renta se asocia con las energías originarias e indestructibles del suelo; la otra parte se pagaría por el uso del capital empleado para mejorar la calidad de la tierra, y para erigir los edi- ficios que se van necesitando con objeto de obtener y conservar el producto"2. De la anterior afirmación se entiende que la renta es muy distinta de los rendimientos que resultan como producto de las mejoras de capital en la tierra. Por tanto, es claro el papel de la acumulación de capital en el crecimiento económico como ya se había anotado antes. Sin acumulación de capital no hay posibilidad de generar los beneficios necesarios para el mayor progreso de la sociedad.

Para Ricardo $(1993,53)$ cuando un país es habitado por primera vez, es decir en el inicio de su colonización, existe abundancia de tierras muy fértiles, por lo que hay que cultivar poco para sostener la población. En esta situación no existe la renta ya que nadie paga por el uso de la tierra. Lo que significa, interpretando a Ricardo, que la tierra es un bien gratuito ${ }^{3}$. En la medida que todos dispongan de tierras para abastecerse del producto necesario para su sustento no habrá lugar al pago de compensación alguna. Según este autor, en esta situación la tierra tiene la misma condición que el agua o el aire, $\mathrm{u}$ otro recurso que sea ofrecido por la naturaleza en cantidades ilimitadas, en términos de que pueden ser libremente obtenidos por los individuos. Así, una primera consideración que debe hacerse en este punto, es el carácter de ilimitado que le da Ricardo a la tierra, tanto como a otros recursos que ofrece la naturaleza. En este primer estadio del desarrollo de los países, para Ricardo $(1993,53)$ la tierra y muchos otros recursos naturales tienen un precio igual a cero, es decir, son bienes gratuitos y por tanto bienes no económicos.

Ricardo $(1993,53)$ plantea que "la tierra no es el único agente de la naturaleza que tiene aptitudes productivas pero es el único o casi el único en el que un conjunto de hombres puede apropiarse para ellos, excluyendo a los demás, apropiándose, por tanto, los beneficios". De esta afirmación puede inferirse, en primer lugar, que los agentes de la naturaleza (sus componentes) poseen las características de

\footnotetext{
2 Según Schuldt $(2004,2)$ Ricardo distingue entre la renta de la tierra "en bruto", que se deriva de sus "energías originarias de renta" de lo que algunos economistas denominan las "rentas de eficiencia" que se atribuyen a las "mejoras" que hacen los que la cultivan, las cuales sí corresponden -como utilidades propiamente dichas- al operario de las tierras o de las minas.

3 Este es el caso de las primeras sociedades en donde no existe aún la propiedad privada.
} 
los bienes públicos puros ${ }^{4}$. No obstante, la tierra, a pesar de ser uno de esos componentes, es un bien público impuro, por lo menos en los primeros estadios del desarrollo, pues sólo posee la característica de no rivalidad en el consumo pero tiene el carácter de exclusión, pues al ser apropiada por un individuo los beneficios derivados de su utilización productiva son sólo de dicho individuo.

Sin embargo, para Ricardo $(1993,56)$ con el progreso de la sociedad y, por tanto, con el crecimiento de la población, se empiezan a utilizar tierras de menor fertilidad a las inicialmente colonizadas y cultivadas, con lo cual aparece la renta en las primeras tierras. La magnitud de esta renta dependerá de la diferencia en las calidades o fuerzas productivas entre estos dos tipos de tierras. Este proceso se repetirá en la medida que siga aumentado la población, es decir, cada vez se utilizará tierra menos fértil con mayor utilización de trabajo lo que hará que aparezca la renta en las tierras anteriormente cultivadas debido a que los precios de los alimentos tendrán que subir para cubrir el costo del trabajo adicional que se aplica en la tierra menos fértil.

De la teoría de la renta de Ricardo surgen interesantes planteamientos para el análisis económico de la utilización del medio ambiente. Este modelo establece el proceso mediante el cual la población presiona a la sociedad a extender la actividad agrícola en áreas que no se utilizaban y, además, muestra la forma como el aumento de los precios de los alimentos llevan a una utilización intensiva de capital y de trabajo. Según Costanza et al. (1999, 30), esto se traduce en la agricultura moderna en mayor utilización de fertilizantes y plaguicidas en tierras cultivadas de buenas calidades. Igualmente, el modelo ricardiano de la renta es importante para comprender la inte- rrelación entre el hombre y la naturaleza ya que en la medida que muestra cómo funciona la relación causal entre crecimiento de población, aumentos de precios de los alimentos con la utilización de la tierra da una idea de la interrelación existente entre las necesidades y deseos humanos y los ecosistemas ecológicos críticos o ecosistemas sustentadores de vida (Costanza et al., 1999, 30).

En este mismo orden de ideas, el análisis económico de Ricardo sobre el uso de la tierra puede vincularse al pensamiento existente en las ciencias de la tierra sobre el uso de los recursos naturales no renovables, pues es una idea común entre los expertos en recursos minerales que los recursos de la mejor calidad se utilizan primero, a pesar de que muchos de dichos recursos no son descubiertos hasta que los recursos de menor calidad ya hayan sido utilizados (Costanza et al. 1999, 32). Sin embargo, un vínculo más estrecho entre la teoría de Ricardo y la ciencia económica contemporánea se encuentra en la economía de los recursos naturales no renovables.

Desde esta área de la economía se plantea que la renta de los recursos es el total de la renta diferencial y la renta de escasez o costo de usuario). La renta diferencial es la asociada al grado de productividad de la tierra, denominada renta ricardiana pues se basa en los principios económicos establecidos por Ricardo para el surgimiento de la renta ${ }^{5}$. La economía de los recursos agotables plantea que las diversas minas, al igual que las tierras destinadas a la agricultura, evidencian diferentes grados de productividad y de costos de explotación. Lo anterior depende, entre otras cosas, de la pureza del mineral, de las características geológicas del mismo, del acceso a infraestructura y otro tipo de costos, que afectan los costos de extracción (Mendoza, 2011, 17).

4 Según Benegas-Lynch $(1998,204)$ "La idea de bienes públicos ha estado implícita en la literatura económica desde Knut Wicksell en adelante, pero contemporáneamente fue Paul Samuelson quien sistematizó la idea de bienes de consumo colectivo o bienes públicos y las consecuentes externalidades (concepto originalmente expuesto por Alfred Marshall y Arthur Cecil Pigou)". Se define como bienes públicos aquellos bienes donde no es posible excluir a nadie de su consumo (principio de no exclusión) y donde la cantidad que un individuo consume de dicho bien no reduce la cantidad disponible para otros individuos (Pindyck \& Rubinfeld, 2001). Lo anterior significa que el costo en el margen de provisión a un consumidor adicional es cero y que no es posible impedir a nadie que lo consuma.

5 La renta de escasez está asociada a la no renovabilidad de los recursos mineros, la llamada "renta de Hotelling". Explotar los recursos mineros (en especial en minas consideradas como menos productivas) tiene un costo de oportunidad: al ser un recurso no renovable, explotarlo hoy implica que no puede ser explotado en el futuro (Mendoza, 2011, 17). 
Así, siguiendo a Ricardo (1993, 57), puede decirse que la aplicación del concepto de renta en la minería contemporánea es similar al aplicado en agricultura. No obstante, se deben establecer algunas consideraciones particulares con relación al concepto original de renta en la agricultura establecido por Ricardo. En particular, la renta minera moderna está vinculada con la ley del mineral que se extrae, la cual no es siempre igual entre minas. Por tanto, podría llegar a existir diversas magnitudes de rentas y, con ello habrían minas que tendrían que pagar un valor de regalías mayor que otras (Pereira, Ulloa, O'Ryan \& De Miguel, 2009, 13). Lo anterior fue resumido por Ricardo $(1993,64)$ quien indica que:

\section{“(...) hay minas de distintas calidades, que entregan resultados muy diferentes, con iguales cantidades de trabajo. El metal ex- traído de la mina más pobre, debe por lo menos tener un valor, no sólo suficiente para adquirir la vestimenta, alimentos $y$ otros artículos necesarios consumidos por los trabajadores, y para llevar el producto al mercado, sino también para pagar las utili- dades comunes y ordinarias a quien antici- pa el capital necesario para llevar a cabo la actividad. El retorno al capital de las minas más pobres que no pagan renta, regularía la renta de las otras minas más producti- vas. Se asume que esta mina rinde las uti- lidades usuales del capital. Todo lo que las otras minas produzcan sobre ese nivel, se pagará necesariamente a sus propietarios como renta".}

Hoy el concepto de renta de Ricardo sigue siendo el más utilizado. No obstante, debe resaltarse que otros autores han adicionado conceptos relevantes a esta definición. En particular, Hotelling (1931, 142) indica que el agotamiento de los recursos naturales genera una renta que se manifiesta en el tiempo. Esta renta vinculada con el agotamiento es la que se conoce como renta de escasez o renta de Hotelling
(Mendoza, 2011, 17). Esta renta de escasez se origina en el hecho de que la decisión de extracción debe considerar, además de sus costos de producción, los costos de oportunidad. Estos últimos se relacionan con la decisión intertemporal de extraer el recurso versus la alternativa de no extraerlo y por ello obtener una renta debido a que el recurso se hace escaso en el tiempo ${ }^{6}$.

\subsection{Una discusión sobre la renta ricardiana y los recursos agotables}

Se plantea que los economistas posteriores han interpretado el criterio de Ricardo acerca de "las fuerzas indestructibles de la tierra" con más rigor del que tuvo Ricardo (Gray, 1914). Pero después de pasar por un proceso de refinamiento gradual a lo largo del tiempo, la definición de renta de Ricardo (1993) se ha reducido a su forma más extrema: en una conclusión de manual de economía donde se plasma que la propiedad de la extensión es la cualidad esencial que distingue a la tierra de otros tipos de mercancías y constituye la base de renta. Así, el concepto de la tierra como base de la renta se ha reducido gradualmente a una mera abstracción. Desde una visión empírica cabría preguntarse por qué es necesario el desarrollo de una doctrina elaborada y particular para explicar el valor de los servicios de los agentes naturales (los ecosistemas) cuando por un supuesto simplificador muchos de esos agentes están excluidos del ámbito de la explicación.

Al respecto, siguiendo a Gray $(1914,467)$, se resalta un interrogante ¿Por qué la renta debe ser un pago por una propiedad original e indestructible para ser renta? La pregunta es parte de la antigua controversia sobre la conveniencia de distinguir la tierra del capital y de diferenciar la renta de otras formas de ingreso. Esta pregunta no será necesaria si se puede demostrar que la indestructibilidad no es una característica que separa la renta de otras formas de ingresos. La razón será entonces evidente para una reconsideración de la teoría de la renta bajo el supuesto de

6 Los costos de oportunidad son intertemporales porque los minerales son recursos naturales no renovables, por lo que explotarlos hoy implica que no pueden ser explotados en el futuro. Sin embargo, en esta teoría no se asume que las reservas aumentarán en el futuro o que existirían cambios tecnológicos por lo que estas rentas estarían sobreestimadas. 
agotabilidad, característica central de muchos de los recursos naturales no renovables, y aún de algunos renovables.

En un sentido, no existe una base o fuente de la renta que sea no perecedera, ya que no hay una fuente concebible que no pierda utilidad con su uso y, por tanto, no pierda su capacidad para producir una renta. Un cambio en la demanda de la sociedad puede causar incluso que la propiedad de extensión pierda la capacidad de generar una renta (Gray, 1914, 467).

Sin embargo, puede discutirse, "con justicia" según Gray (1914, 467), que la palabra indestructible ha sido empleada por Ricardo y sus seguidores en todo otro sentido: en el sentido que el uso por el cual la renta es pagada no causa el deterioro de la fuente de renta. Bajo este entorno la fuente de la renta puede ser indestructible. Gray $(1914,468)$ establece que el ejemplo más claro de esta situación es el suelo urbano. En este caso es la demanda social la que induce a que la renta no disminuya sino que, incluso, llegue a aumentar. Así, es claro que es la dinámica en los cambios de uso de la tierra la que llega a afectar la renta asociada a un uso u otro.

Ahora bien, en el caso de tierras dedicadas a la agricultura también con frecuencia es posible aislar los ingresos atribuibles a las propiedades indestructibles. Cuando el recurso natural que se agota tiene sustitutos, el costo de su sustitución determina el valor del recurso agotado ${ }^{7}$; y el resto del excedente total se puede considerar la renta pagada por las propiedades inagotables. En muchos casos, sin embargo, no es posible aislar los retornos asignables a las propiedades indestructibles. Como un ejemplo, en las minas es imposible separar el valor de las propiedades que agotadas del valor de las propiedades inagotables. Por tanto, se observa que bajo el supuesto de Ricardo la renta se vincula con una parte del universo de los recursos naturales existentes. Además, como ya se planteó antes, es muy difícil, sino imposible, distinguir la renta de los ingresos proveniente de los elementos destructibles. Para Gray (1914, 469)
Estos hechos parecen justificar el intento de alterar el concepto Ricardiano de la renta de forma tal que se evite la necesidad de utilizar en el análisis el supuesto de que la renta es pagada sólo por la cualidad de no destrucción del suelo.

En este punto es importante analizar lo que significa agotamiento. Al respecto Gray (1914) lo define como un cambio de lugar o un cambio en la forma del recurso en cuestión. Un mineral como el oro puede ser removido de una mina y continuar no destruido, es decir permanecer en el tiempo para su uso. En tal caso, al agotamiento se le relaciona la movilidad del recurso con respecto a un sitio dado. En cuanto a la teoría de la renta, el agotamiento, con referencia a una localidad en particular es el que importa si los elementos valiosos están absolutamente consumidos o simplemente trasladados desde el sitio original hacia otro lugar. Sin embargo, este agotamiento puede evitarse o mitigarse restaurando otros recursos de la misma clase en lugar de aquél recurso que es extraído y utilizado en el proceso económico.

No obstante, esto dependerá de las condiciones económicas que rodean el proceso de sustitución del recurso en cuestión, básicamente de las condiciones físicas del sustituto y de la tecnología utilizada. Es decir, tal tipo de no agotamiento dependerá de la viabilidad económica de la sustitución del recurso. Incluso en el caso de la agricultura, la experiencia general en el mundo ha demostrado que la restauración es, con frecuencia, poco rentable. En este sentido, el agotamiento puede ser una característica, bajo ciertas condiciones, de muchos de los recursos naturales, incluyendo muchos de los que son recursos renovables (Gray, 1914; Harwick, 1997).

La relación del supuesto del agotamiento con la teoría de la renta depende en gran medida de la posibilidad de evitar el agotamiento con respecto a una localidad dada. Gray $(1914,469)$ divide el análisis en este contexto en dos casos: i) cuando la prevención del agotamiento es económicamente viable y, ii) cuando dicha prevención no es económicamente viable. Para el primer caso, se plantea que prevenir

7 Este tipo de pago se denomina "costo de usuario" (Riera, 2005). 
el agotamiento puede hacerse con o sin gasto adicional. Las condiciones económicas particulares señalarán la ocurrencia de una u otra situación. Cuando la prevención no requiere un incremento adicional de recursos económicos la teoría ricardiana no es invalidada por el supuesto de agotamiento, ya que todo el retorno económico atribuible a la tierra es un excedente que se acumula siempre que las condiciones de oferta y demanda se mantengan inalteradas. El agotamiento se produce, pero el proceso de restauración es marginal al proceso de la mayor utilización económica. De otro lado, en los casos en los que es imposible o no es rentable la prevención de agotamiento, se requeriría un ajuste de la doctrina de la renta si se quiere evitar utilizar el supuesto de la inagotabilidad (Gray, 1914, 470).

Siguiendo a Gray (1914), como una conclusión de esta relación renta ricardiana y recursos naturales agotables, se establece que bajo el supuesto de la agotabilidad la tierra se asemeja al factor trabajo en el sentido de que perece no por el uso sino por la falta de uso. $\mathrm{Si}$ es capaz de proporcionar un servicio económico periodo a periodo, el hecho de no utilizarlo en cualquier periodo es el origen de la pérdida, así como la mano de obra sufre pérdida al quedar desempleada. Sin embargo, cuando se asume que los beneficios -es decir, el flujo de servicios ambientales, como se conocen en la literatura de la economía ambiental- que pueden derivarse de un recurso natural son no sustituibles o agotables, el punto de vista se altera.

Como ejemplo, Gray $(1914,470)$ plantea un propietario de un yacimiento de un mineral de alto valor que desea obtener el máximo beneficio de un stock del recurso en cuestión (oferta limitada). Si por cualquier razón el propietario obtiene un menor beneficio de la extracción y venta del mineral en el periodo presente del que obtendría en un periodo futuro, entonces podría ser rentable posponer la extracción y utilización del mineral. Si bien el anterior es fruto de una concepción modificada de lo que es renta con respecto al enfoque ricardiano, es claro que la visión de Ricardo con relación a la característica de indes- tructibilidad del recurso suelo ha sido fuente de inspiración para analizar las características particulares y los tipos de rentas asociadas a los recursos naturales con relación a la agotabilidad económica o de localización, a lo largo del proceso de consolidación del área de la economía de los recursos agotables, cuyos resultados teóricos más destacados se evidencian en los trabajos de Gray (1914), Hotelling (1931) y Hartwick $(1977 ; 1997)$.

\section{Ricardo y los rendimientos decrecientes}

De otra parte, ligado al problema de la renta de la tierra en Ricardo, se encuentra lo relacionado a los rendimientos del recurso. Para Ricardo $(1993,65)$, la causa de los rendimientos decrecientes de la tierra es el número de la población. Este autor observó que, en Inglaterra, progresivamente se hacía un uso intensivo de la tierra. Además, planteaba que sus productos agrícolas, en innumerables ocasiones, no estaban en las mismas proporciones que las cantidades adicionales de los recursos capital y trabajo. Desde la óptica de Ricardo, esta ley podría plantearse como sigue: si a una cantidad fija de tierra se agrega dosis recurrentes de trabajo, en un principio el rendimiento será más que proporcional, y por último menos que proporcional. En otras palabras se puede establecer que más trabajo y más gastos aplicados a la tierra no tienen un beneficio equivalente. Así, desde la literatura económica tradicional se plantea que Ricardo es el precursor de lo que desde la teoría neoclásica se conoce como rendimientos marginales decrecientes ${ }^{8}$.

Ahora bien, Huerta $(2001,74)$ afirma que "aunque Malthus y Ricardo tradicionalmente han sido identificados en la literatura como los primeros en plantear la ley de los rendimientos decrecientes, en realidad, según Birmingham, el primer enunciado claro de esta ley fue realizado por Turgot en el siglo XVIII". Birmingham $(1978,58)$ plantea que en el año 1815 se publicaron cuatro ensayos, cada uno vinculado con un autor (West, Torrens, Malthus y Ricardo), donde se hace mención sobre la renta diferencial de

8 No obstante, Samuelson \& Nordhaus (2002, 97) plantean que “(...) la ley de los rendimientos decrecientes es una regularidad empírica ampliamente observada más que una verdad universal como la ley de la gravedad. Se ha encontrado en numerosos estudios, pero también se han descubierto excepciones". 
la tierra como consecuencia de los altos precios de los granos. Según Birmingham $(1978,58)$, la explicación última dada en los cuatro ensayos acerca de la aparición de altos precios y rentas diferenciales es el principio de los rendimientos decrecientes.

Ahora, Blaug $(2001,112)$ plantea que los ensayos en cuestión presentaban en común tres particularidades de la ley de los rendimientos decrecientes: i) pensaban que dicha ley sólo se aplicaba a la agricultura; ii) Era válida para el mediano y largo plazo a pesar del surgimiento del cambio técnico; y iii) era producto de la observación de la vida económica del campo. Según Blaug $(2001,112)$, la mayoría de los economistas clásicos consideraban la ley de los rendimientos decrecientes como una simple generalización de las experiencias comunes; mientras que los economistas modernos definen dicha ley como una afirmación de lo que ocurre si se aumenta la cantidad de un insumo, ceteris paribus los demás. Para Blaug $(2001,113)$ la definición moderna de la ley de los rendimientos decrecientes no puede probarse con un vistazo al mundo real.

Huerta $(2001,74)$ anota que es importante establecer que la teoría de Ricardo parte de suponer que los terrenos utilizados en la agricultura son de una extensión definida en cualquier país, por lo que sus linderos no podían ampliarse. Así, argumenta Huerta $(2001,74)$ que con base a esa mirada Ricardo considero que si a esa cantidad fija de tierras se le añadía trabajo una y otra vez, a causa del crecimiento de la población, este proceso llevaría a que, al adicionar un trabajador más, el producto agrícola no mejoraría, es decir que el producto adicional obtenido con una unidad más de trabajo sería menor que el obtenido con la unidad de trabajo anterior. Lo anterior, en términos de la teoría económica moderna significa que la productividad marginal del trabajo sería decreciente, luego de cierta cantidad de dicho factor productivo.

Sin embargo, la preocupación de Ricardo va más allá de la relación técnica entre insumos y producción ya que, según Huerta (2001, 75), este buscaba una explicación general del origen de la renta de la tierra, es decir, de un fenómeno económico real que afectaba a la sociedad, en particular de un problema de la
Inglaterra de su época. Así, desde la teoría económica de Ricardo se establece que el producto marginal del trabajo que se aplica en la actividad agrícola es el que determina el precio de los alimentos. Cuando se presentan los rendimientos decrecientes por la mayor utilización de las tierras hasta llegar hasta las tierras de menor fertilidad debido al crecimiento de la población, el producto agrícola obtenido en las tierras menos fértiles servirá para fijar el precio, el cual será mayor que el que se da en las tierras más productivas. De esta forma el bien se vende a ese precio alto y quienes tienen una mayor productividad en sus tierras obtendrán una renta diferencial mayor.

Siguiendo a Huerta $(2001,75)$, se puede afirmar que la explicación de Ricardo $(1993,70)$ al fenómeno de los rendimientos decrecientes es una explicación a un problema creciente en su época: el acelerado crecimiento de la población y la posibilidad de no llegar a proveer la producción adecuada de alimentos, a partir de una cantidad fija de tierra, que respondiera a una demanda de bienes cada vez mayor, y a un precio que ya no generara renta a los propietarios de las tierras. Este problema se vinculaba pues con rendimientos decrecientes en el mediano y largo plazo a causa de unas cantidades fijas de tierra y, por tanto, terminaba por impactar a los ingresos de los terratenientes (Huerta, 2001, 75).

Al respecto Cannan $(1942,188)$ afirma que dicha ley es pseudo histórica y pseudo científica, y que no está de acuerdo con la verdad histórica. Además plantea que "desde Estados Unidos, H. C. Carey se mostró en contra de la teoría Malthus-ricardiana, afirmando que «la experiencia demostraba que los aumentos de la población eran favorables a la mayor productividad de la industria»" (Cannan, 1942, 190). Con base en esto, Cannan $(1942,192)$ afirma que la ley de los rendimientos decrecientes no tiene demostración histórica, ni sustento lógico, aún en el sector agrícola.

\section{Una síntesis de la teoría económica ricardiana: puntos críticos en torno a los rendimientos decrecientes de la tierra y el problema poblacional}

Desde la literatura algunos autores afirman que la obra de David Ricardo de cierta forma fue un análi- 
sis crítico a la riqueza de las naciones de Adam Smith (Hahne-Rima, 1995, 145). Sin embargo, lo que si es cierto es que el análisis económico de Ricardo ofreció una perspectiva más amplia que la de su predecesor a una ciencia naciente como lo era la economía política para la época. Un paso relevante para la ciencia económica dado por Ricardo fue el establecimiento del primer modelo económico formal. Con dicho modelo llegó a obtener relevantes resultados sobre el comportamiento de las variables económicas, que se convirtieron luego en bases fundamentales de la teoría económica moderna.

A pesar de que el principal interés de Ricardo era la división del producto de la nación entre las tres principales clase sociales en la forma de salarios, beneficio y renta, un punto central de su modelo económico, y en el cual el rol de la tierra es crucial, se encontraba en la creencia de que el crecimiento económico se detendría antes o después, debido al costo creciente de producir alimentos cuando la tierra disponible era limitada (Hahne-Rima, 1995, $146)^{9}$. Un razonamiento importante detrás de esta conclusión era el principio malthusiano, formulado en el "Ensayo sobre el principio de la población" escrito por Thomas Malthus en 1798, que plantea que la población tiende a crecer de forma constante hasta los límites que presenta la oferta disponible de alimentos (Hahne-Rima, 1995).

Para Ricardo (1993), a medida que crece la fuerza laboral sólo se puede incrementar la producción agrícola, para satisfacer la demanda creciente de alimentos, ya sea ampliando la frontera agrícola hasta llegar a utilizar las tierras menos fértiles o utilizando más cantidad de trabajo y de capital en las tierras cultivadas. Sólo así se aumentarían las cosechas. Aunque los salarios van a disminuir, los beneficios no aumentarían en la misma proporción ya que los agricultores competirían entre sí por cultivar las mejores tierras. Dado este proceso, según Ricardo (1993, 73), los propietarios de tierras son quienes más se beneficiarían del progreso económico. En este orden de ideas, para este autor el problema son los rendimientos decrecientes de la tierra.
Por tanto, su propuesta para solucionar esta situación es la importación de alimentos desde otros países. En este sentido, es importante resaltar la libertad para dicha importación desde países que tuvieran una ventaja comparativa en el costo del trabajo como el medio más efectivo de disminuir la presión del costo creciente de los granos, el cual está detrás de las participaciones del ingreso a largo plazo (Hahane-Rima, 1995, 171). Desde la perspectiva del análisis económico de la tierra como un recursos naturales se plantea que, al igual que la tierra los recursos naturales tienen una oferta limitada; aún en el caso de recursos naturales renovables el aumento de su oferta está depende que su tasa de regeneración este por encima de su tasa de utilización.

Ahora bien, su tasa de crecimiento será negativa debido tanto al crecimiento de la población como a factores de mercado, como es el caso de expectativas de precios bajos para el recurso en el futuro con respecto al precio vigente en el mercado. Así, en muchos recursos naturales el problema no serán los rendimientos decrecientes en su utilización, como sí ocurre en el caso de la tierra, sino un problema de sobre utilización por encima de la capacidad de regeneración biológica del recurso, en donde la utilización de capital (maquinas) aceleraría la utilización del recurso. A pesar de todo, la idea la detención del crecimiento económico por factores de productividad de la tierra y presión poblacional, el llamado estado estacionario de la economía, es un elemento importante como punto de partida del análisis económico del medio ambiente.

De otro lado, siguiendo a Hahne-Rima (1995, 171), este afirma que la renta, desde la óptica de David Ricardo, sólo se aplica a la tierra en conjunto (no hay separabilidad entre la característica destructible y la indestructible del suelo) ya que no existe un precio de oferta de la tierra que deba ser satisfecho para garantizar su producción. No obstante, como la tierra tiene usos competitivos, ésta tiende a utilizarse en el uso que sea más productivo, por lo que se requerirá un pago por su escasez en el uso alternativo. Este pago es lo que se define en la teoría económica mo-

9 De hecho, para Blaug $(2001,109)$ “(...) en el centro del sistema ricardiano se encuentra la noción de que el crecimiento económico debe terminar tarde o temprano debido a la escasez de recursos naturales". 
derna como costo de oportunidad, que es un factor que determina el precio, al igual que el costo de la mano de obra (trabajo) o el costo del capital. Dicho precio hoy se conoce como "precio de transferencia" del agente (Hahne-Rima, 1995, 171) ${ }^{10}$.

Desde la teoría económica moderna, no hay inclinación especial por alguna teoría en especial de la renta de la tierra. Hoy es claro que la tierra no es un regalo de la naturaleza y que requiere la inversión de recursos económicos de inversión y operación. Además, hay un reconocimiento de la escasez (agotabilidad) de los recursos naturales y el medio ambiente en general. Por tanto, la tierra toma el papel de un factor productivo más, al igual que el capital y la mano de obra, a pesar de su mayor inelasticidad en la oferta con relación a la elasticidad de los otros factores productivos.

\section{Conclusiones}

Dentro del pensamiento clásico, el análisis de la renta de la tierra de Ricardo es uno de los aportes relevantes de este autor, dado su exposición sistemática y rigurosa. Además, se destaca que desde la teoría de la renta ricardiana se desprenden argumentos importantes para el análisis del medio ambiente y los recursos naturales con relación a la presión que ejerce el aumento de la población sobre la utilización cada vez mayor de la naturaleza y con ello, el deterioro futuro que puede generarse en la tierra, vía fertilizantes y pesticidas, gracias a la intensificación de la agricultura.

Ahora, un punto central en el modelo económico de Ricardo, donde la tierra toma un papel preponderante, se encuentra en la creencia de que el crecimiento económico se detendría en algún momento (el llamado estado estacionario) debido al costo incrementado de producir alimentos con crecientes limitaciones de tierra disponible. Detrás de este argumento está el principio malthusiano que declara que las tasas de crecimiento de la población superan al crecimiento decreciente de la oferta de alimentos, debido, de un lado, a las limitaciones que imponen los rendimientos decreciente de la tierra y, por otro lado, por una tasa de cambio tecnológico que no va al ritmo del crecimiento de la población.

Un aspecto relevante de la renta ricardiana es que está asociada a una sola clase de agotamiento: el agotamiento por localización o movilidad del recurso. Sin embargo, se plantea que el agotamiento por localización es el que importa si los elementos valiosos están absolutamente consumidos o simplemente trasladados desde el sitio original hacia otro lugar. En este punto, desde la economía de los recursos naturales se argumenta que el enfoque ricardiano es parcial pues no percibió la posibilidad real de que este agotamiento puede evitarse o mitigarse restaurando otros recursos de la misma clase en lugar de aquél recurso que es extraído y utilizado en el proceso económico. Este elemento de análisis es conocido en la literatura como elasticidad de sustitución del recurso. No obstante, éste dependerá de las condiciones económicas que rodean el proceso de sustitución del recurso en cuestión, básicamente de las condiciones de las condiciones físicas del sustituto y de la tecnología utilizada.

De otro lado, se plantea que el enfoque de la renta ricardiana es válido cuando la prevención de agotamiento no requiere un incremento adicional de recursos pues toda la renta atribuible a la tierra es un excedente que se acumula siempre que las condiciones de oferta y demanda no cambien. En este contexto el agotamiento se produce, pero el proceso de restauración del recurso se exógeno al proceso de la mayor utilización económica del recurso. En cambio, se afirma que en las situaciones de no sustitución física del recursos o en caso de inviabilidad económica de la prevención de agotamiento, se requiere de un ajuste de la doctrina la renta ricardiana ya que desaparece el supuesto de la indestructibilidad del recurso tierra.

10 Hahne-Rima $(1995,172)$ establece “(...) que en el ámbito de la empresa individual que adquiere un factor productivo, los precios de transferencia hacen parte de los costos de producción, aun cuando sean superávit desde la perspectiva de la industria o de la economía de una nación, en el sentido que su eliminación no disminuye la oferta de dicho factor". Ahora, solo en la situación en la que los servicios de un factor, en este caso la tierra, están limitados a una única opción de uso se considera que todo el pago es renta, tanto desde una perspectiva individual como social, porque su precio de transferencia es cero. 
Para terminar, se afirma que la naturaleza, bajo un enfoque de agotabilidad por localización, ha estado presente en la teoría económica propuesta por Ricardo. En el trabajo de este autor a la tierra se la identifica explícitamente como fuente de riqueza, ya que, según el enfoque de Ricardo, sólo la combinación de las "fuerzas indestructibles de la tierra" en conjunto con el trabajo es la que hace posible la generación de valor $y$, por lo tanto, de riqueza para la sociedad.

No obstante, hoy en día en la economía ambiental se reconoce el aporte a la riqueza (y con ello al bienestar social) de la naturaleza incluida la tierra) independiente de la aplicación o no de trabajo (capital humanos) en virtud de la característica de no sustitución física o de su criticidad en términos ecológicos, económicos y sociales, lo cual implica una revisión del concepto de renta ricaridiano y en donde cobra importancia el concepto de costo de oportunidad o costo de usuario. A pesar de todo, la teoría de la renta diferencial de Ricardo, ha aportado elementos de análisis relevantes para la economía moderna de los recursos naturales que ha emergido desde principios del siglo XX.

\section{Referencias}

Alfranca, O. (2012). Evolución del pensamiento económico sobre los recursos naturales. En: Nuevas Corrientes del Pensamiento Económico, ICE, N 865 , marzo-abril: 79-90.

Benegas-Lynch, A. (1998). Bienes públicos, externalidades y los free-riders: el argumento reconsiderado. En: Estudios Políticos, $\mathrm{N}^{\circ} 71:$ 203-218.

Birmingham, W. (1978). Introducción a la economía. México. Siglo XXI Editores.
Blaug, M. (2001). Teoría económica en retrospección. México. Fondo de Cultura Económica.

Cannan, E. (1942). Historia de la teoría de la producción y la distribución en la economía política inglesa. México. Fondo de Cultura Económica.

Costanza, R., Cumberland, J., Daly, H., Goodland, R. \& Noorgard, R. (1999). Una introducción a la economía ecológica. México. Editorial Cecsa.

Gray, L. (1914). Rent under assumption of exhaustibility. En: Quarterly Journal of Economics, 28: 466-489.

Hahne-Rima, I. (1995). Desarrollo del análisis económico Quinta edición. Madrid. Editorial Irwin.

Hartwick, J. (1977). Intergenerational equity and the investing of rents form exhaustible resources. En: American Economic Review, 67(5): 972-974.

Hartwick, J. (1997). The economics of natural resource use. Massachusetts. Addison Wesley Publishers.

Hotelling, H. (1931). The economics of exhaustible resources. En: Journal of Political Economy, (39): 137-175.

Huerta, R. (2001). De nuevo los rendimientos decrecientes. En: Aportes, 6(18): 73-90.

Mendoza, W. (2011). La política impositiva aplicable a los minerales y al petróleo: teoría, experiencias y propuesta de política para el Perú, Lima. Pontificia Universidad Católica del Perú. Consorcio de investigación económica y social (CIES).

Pearce, D. (2002). An intelectual history of environmental economics. En: Annual Review of Energy and the Environment, Palo Alto, (27): 57-81.

Pereira, M., Ulloa, A., O’Ryan, R. \& De Miguel, C. (2009). Síndrome holandés, regalías mineras y políticas de gobierno para un país dependiente de recursos naturales: el cobre de Chile. En: Medio ambiente y desarrollo. CEPAL. Serie $\mathrm{N}^{\circ} 140$.

Pindyck, R. \& Rubinfeld, D. (2001). Microeconomía. 5² Edición. Madrid. Prentice Hall.

Ricardo, D. (1993). Principios de economía política y tributación. Bogotá. Fondo de Cultura Económica.

Riera, P. (2005). Manual de economía ambiental y de los recursos naturales. Madrid. Thomson Editores.

Samuelson, P. \& Nordhaus, W. (2002). Microeconomía, Décimo séptima edición. México. Mc Graw Hill.

Schuldt, J. (2004). Regalías mineras y rentas ricardianas. SUNAT, 2013. Disponible en: www.sunat.gob.pe 